\title{
Research on the Influencing Factors of Co-working Space Development --A Case Study of Lvliang, Shanxi Province
}

\author{
Ziming Zhao ${ }^{1}$, Jialin Wang ${ }^{1}$, Siyuan Hou ${ }^{2}$ \\ ${ }^{1}$ Statistic Institute, Shanxi University of Finance and Economics, Wucheng road, Taiyuan, China \\ ${ }^{2}$ Financial and Monetary Institute, Shanxi University of Finance and Economics, Wucheng road, \\ Taiyuan, China
}

Keywords: co-working space, factor analysis, regression analysis, empirical analysis

\begin{abstract}
In the initiative of "public entrepreneurship and innovation", co-working space goes into the people's vision, and gradually hatches a large number of small companies. However, in Shanxi, co-working space is also under tremendous pressure to survive. The problems of homogeneity of service resources, inefficient service and high input costs are gradually highlighted. This paper focuses on analyzing the survey data and establishing a reasonable model to explore the causes and influencing factors of the space development in co-working spaces in Shanxi Province. Through the survey data of 10 co-working spaces in Lvliang, Shanxi Province, the empirical analysis is carried out. The data noise is processed and the reliability and validity of the data are obtained. The regression model is established and the factor analysis is carried out. Finally, effective conclusions are drawn and reasonable suggestions are given for the development of co-working space
\end{abstract}

\section{INTRODUCTION}

On March 16, 2016, at the press conference of the Fourth Session of the 12th NPC, State Department Premier Li Keqiang mentioned in his answering a question: It is necessary to combine the cultivation of new kinetic energy with the transformation and upgrading of traditional kinetic energy to form a "double engine". He emphasized: "We advocate public entrepreneurship and create new innovations. In fact, we provide a platform for small, medium and large-sized enterprises, scientific research institutions and other industries to create a space for innovation, crowdsourcing, public support and crowdfunding."

In Shanxi, co-working space has grown rapidly. At the beginning of 2016, the province first identified 35 provincial-level co-working spaces. In addition, dozens of co-working spaces did not pass the provincial-level accreditation. In the province's "Several Opinions on Implementing Scientific and Technological Innovation", it is proposed that the number of public foundations in our province will reach 100 by 2017. The survey found that the frenzied expansion has also put enormous pressure on subsistence for all kinds of conglomerates, with the problems of homogeneity of service resources, ineffective service provision, and high input costs.

\section{SURVEY PIAN}

\subsection{Survey Object}

The survey focuses on Internet Innovation Park and Cultural and Creative Park of Changyuan in Lvliang, Shanxi Province. The investigation mainly focuses on the operation of co-working space and the development status and requirements of internal companies.

\subsection{Quality Control}

\subsubsection{Validity Test}

Validity usually refers to the validity and correctness of the questionnaire. We usually using expert evaluation method to discuss the content validity. For structural validity, we test the validity of the structure by SPSS. KMO value was $0.957>0.9, \mathrm{P}<0.01$, indicating that the validity of the data structure. 


\subsubsection{Reliability Test}

Reliability refers to the consistency of the results obtained when making repeated measurements of the same thing, which reflects the stability, reliability and the trueness of the measured features of the measurement tools. In general, the internal consistency is often used to indicate the level of reliability. Through reliability analysis by SPSS, the Cronbach's alpha coefficient of the questionnaire is 0.988, which shows that the reliability of the questionnaire is high and the reliability is good, so it is suitable for the follow-up factor analysis.

\section{EMPIRICAL ANALYSIS}

\subsection{Data Analysis}

In general, the data collected in the questionnaire are always incomplete. Therefore, the accuracy of data analysis needs to be improved by smoothing the noise data by data processing, adding missing values, and deleting or identifying outliers. Incomplete data mainly for the lack of data and data errors. There are currently two ways to deal with missing data, one is to delete rows with missing data and the other is to replace the missing data with a reasonable replacement value.

\subsection{Statistic Analysis}

Among the scores of satisfaction of service by employees in co-working space, we surveyed with the questionnaire, the result shows:

(1) With respect to co-working space infrastructure, the insiders who settled in the enterprises were more satisfied with the office space, with the evaluation rate reaching $88.46 \%$ above "normal". For the provision of facilities, the average score reached 3.47 and 3.49 , showing that the overall satisfaction of enterprises settled in basic services. With the current actual situation of co-working space in Shanxi Province, as of September 2016, there are 101 provincial co-working spaces in Shanxi Province with a total area of 48.96 million square meters and a total of 17,400 new business incubators, more than 3,500 of teams. In the process of field interviews, we see co-working space settled enterprises spacious and clean office space, each business has its own independent office space. Each floor has an independent central air conditioning and heating facilities, Wi-Fi full floor coverage, to achieve "bag settled". We can see co-working space infrastructure development is better.

(2) In terms of entrepreneurial counseling for co-working space, the results of the questionnaire showed that co-working space did not do well in training, salon, consultation and so on. Some even created a cynical space, and organized large lectures early in the founding but no start-up counseling. The score of this item is only 3.28, 21.54\% below "normal"(Changsheng He,2016).

(3) Regarding the financial support, co-working space can provide financial support and investment and financing channels for the fundamental development and expansion of small enterprises. (Chunqing Wen, Junfang Jin, Xiaoyan Xu,2016) Despite the country's proposal of "public entrepreneurship and innovation", small enterprises are still facing financing difficulties in the project provinces. The results show that in the "provide some financial support for the project (or in-kind support)", the user satisfaction is mainly concentrated in 3 to 4 scores, showing that there is a great demand for business hoping to get more support (Hongyi Dong,2016).

\subsection{Factor Analysis}

The basic idea of factor analysis is to group the independent variables that affect one dependent variable according to the relevant degree of, and then combine the variables to achieve the goal of dimension reduction. The merged variable is called a common factor, and the dependent variable is a linear combination of the common factors.

\subsubsection{Investigate Whether the Original Variable is Suitable for Factor Analysis}

First of all, we should examine whether there is a certain linear relationship between the original variables of the satisfaction of co-working space, whether it is suitable for factor extraction using factor analysis. Here, we analyze the correlation coefficient matrix of variables, reaction-like correlation matrix, Bartlett's test and KMO test method. At the same time, due to the existence of missing values in the data, the missing value was treated with the elimination of variation value.

From the correlation coefficient matrix table of the original variables, we can find that the 
variables show a strong linear relationship, from which public factors can be extracted, suitable for factor analysis.

Table 1:and Bartlett test of sphericity.

\begin{tabular}{|cc|c|}
\hline \multicolumn{1}{|c|}{ KMO test } & .909 \\
& $\chi^{2}$ & 1680.913 \\
Bartlett test of sphericity & $\mathrm{df}$ & 136 \\
& Sig. & .000 \\
\hline
\end{tabular}

The observed value of Bartlett test of sphericity was 1680.913 and the corresponding probability $\mathrm{P}$-value is close to 0 . If the significance level is 0.05 , since the probability P-value is less than the significance level , the original hypothesis should be rejected as the correlation coefficient matrix Significant differences with the unit matrix. In the meantime, the KMO value is 0.909, which is restrained from being suitable for factor analysis according to the KMO metric given by Kaiser.

\subsubsection{Extract the Factor}

Here we conduct a tentative analysis: Based on the correlation coefficient matrix of the original variables, we use principal component analysis to extract the factors and extract eigenvalues greater than one.

Table 2: Common factor variance.

\begin{tabular}{|c|c|c|}
\hline & & \\
\hline A & 1.000 & .777 \\
B & 1.000 & .563 \\
C & 1.000 & .510 \\
D & 1.000 & .577 \\
E & 1.000 & .455 \\
F & 1.000 & .589 \\
G & 1.000 & .656 \\
H & 1.000 & .540 \\
I & 1.000 & .441 \\
J & 1.000 & .525 \\
K & 1.000 & .654 \\
L & 1.000 & .597 \\
M & 1.000 & .721 \\
N & 1.000 & .640 \\
O & 1.000 & .604 \\
P & 1.000 & .626 \\
Q & 1.000 & .671 \\
\hline
\end{tabular}

From the table, the first column of data is the commonality of variables under the initial solution of the factor analysis. It shows that if all the eigenvalues are extracted from the original 17 variables by using the principal component analysis, all the variances of the original variables can be explained, the commonality of all variables are 1 . In fact, the number of factors is less than the number of the original variables, which is the target of the factor analysis. All the eigenvalues cannot be extracted. The second column of data is the common degree of the variables when extracting the eigenvalues from the specified extraction conditions, where the eigenvalue is greater than 1. It can be seen that most of the information on the satisfaction factors of co-working Space can be explained by most of the factors, and there is less information about the loss of these variables. The overall effect of this factor extraction is generally clear. 
Table 3: Total variance explained.

\begin{tabular}{|c|c|c|c|c|c|c|c|c|c|}
\hline \multirow{2}{*}{ Ingredients } & \multicolumn{3}{|c|}{ Initial eigenvalue } & \multicolumn{3}{c|}{ Extract square and load } & \multicolumn{3}{c|}{ Rotate squared and load } \\
\cline { 2 - 9 } & total & variance & Accumulative & total & variance & Accumulative & total & variance & Accumulative \\
\hline 1 & 7.954 & 46.791 & 46.791 & 7.95 & 46.791 & 46.791 & 3.724 & 21.905 & 21.905 \\
2 & 1.180 & 6.939 & 53.730 & 1.18 & 6.939 & 53.730 & 3.723 & 21.902 & 43.807 \\
3 & 1.013 & 5.959 & 59.689 & 1.01 & 5.959 & 59.689 & 2.700 & 15.882 & 59.689 \\
4 & .939 & 5.524 & 65.213 & & & & & & \\
5 & .733 & 4.313 & 69.526 & & & & & & \\
6 & .672 & 3.956 & 73.482 & & & & & & \\
7 & .649 & 3.819 & 77.301 & & & & & & \\
8 & .608 & 3.575 & 80.877 & & & & & & \\
9 & .542 & 3.190 & 84.067 & & & & & & \\
10 & .493 & 2.900 & 86.967 & & & & & & \\
11 & .466 & 2.740 & 89.707 & & & & & & \\
12 & .394 & 2.317 & 92.024 & & & & & & \\
13 & .342 & 2.009 & 94.034 & & & & & & \\
14 & .300 & 1.766 & 95.800 & & & & & & \\
15 & .267 & 1.570 & 97.370 & & & & & & \\
16 & .239 & 1.406 & 98.776 & & & & & & \\
17 & .208 & 1.224 & 100.000 & & & & & & \\
\hline
\end{tabular}

From the table we can see that the first column is the factor number, the latter three columns form a group. The meaning of the data items in each group is the eigenvalue, the variance contribution rate and the cumulative variance contribution rate at a time.

The first set of data items (second column - fourth column) describes the initial solution of the factor analysis. The first factor has a characteristic value of 7.954, which accounts for $46.791 \%$ (7.954 /17* $100 \%)$ of the total variance of the original 17 variables, and the cumulative variance contribution rate is $46.791 \%$. The second factor has a characteristic value of 1.180 , explaining the total variance of the original 17 variables of $6.939 \%(1.180 / 17 * 100 \%)$, the cumulative variance contribution rate of $53.730 \%$. The rest of the data has a similar meaning. Since 17 factors are extracted in the initial solution, the total variance of the original variables is explained, and the cumulative variance contribution rate is $100 \%$. The second set of data items ( columns 5 to 7 ) describe the case of factorial levels. We can see that due to the extraction of the three factors, the three factors account for $59.689 \%$ of the total variance of the original variables. In general, the original variable has less information loss and the factor analysis is better.

The third group of data items describes the case of the final factor level. It can be seen that after the factor is rotated, the variance contribution rate of the total cumulative variable does not change much, that is, it does not affect the commonality of the original variable, but redistributes each factor to explain the variance of the original variable and changes the variance of each factor Contributions make the factor easier to explain.

From the figure, the abscissa is the number of factors and the ordinate is the eigenvalue. It can be seen that the first factor has a high eigenvalue and contributes the most to explaining the original variable. The fourth and subsequent factors have small eigenvalues and little contribution to interpreting the original variables. They can already become negligible "gravel at the foot of the mountain", so it is appropriate to extract three factors.

From the table, we show the factor loading matrix, which is the core of factor analysis. According to the table, we can draw the factor analysis model of this application case. 


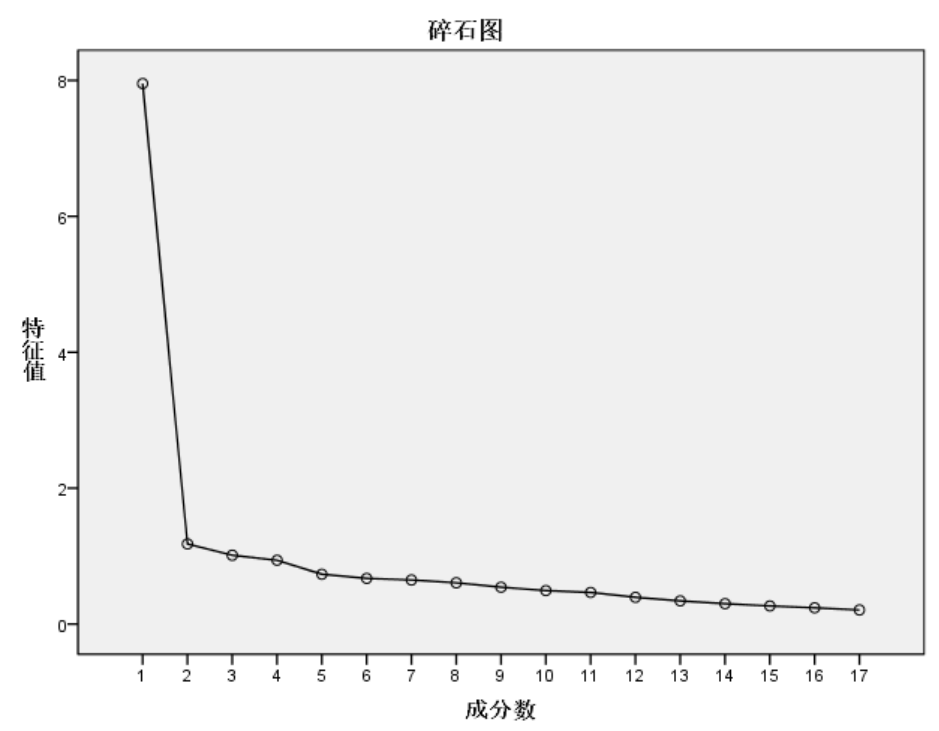

Figure 1: Gravel figure.

Table 4: Component matrix.

\begin{tabular}{|c|c|c|c|}
\hline & \multicolumn{3}{|c|}{ Ingredients } \\
\cline { 2 - 4 } & 1 & 2 & 3 \\
\hline $\mathrm{A}$ & .664 & -.568 & .119 \\
$\mathrm{~B}$ & .679 & -.273 & .168 \\
$\mathrm{C}$ & .706 & -.084 & .072 \\
$\mathrm{D}$ & .744 & -.068 & -.136 \\
$\mathrm{E}$ & .673 & -.054 & -.008 \\
$\mathrm{~F}$ & .688 & .149 & .306 \\
$\mathrm{G}$ & .557 & .568 & .148 \\
$\mathrm{H}$ & .729 & -.072 & -.060 \\
$\mathrm{I}$ & .623 & .180 & -.143 \\
$\mathrm{~J}$ & .718 & -.032 & -.092 \\
$\mathrm{~K}$ & .614 & -.296 & -.435 \\
$\mathrm{~L}$ & .732 & -.194 & .152 \\
$\mathrm{M}$ & .694 & -.101 & .479 \\
$\mathrm{~N}$ & .660 & .340 & .297 \\
$\mathrm{O}$ & .705 & .288 & -.154 \\
$\mathrm{P}$ & .703 & .119 & -.343 \\
$\mathrm{Q}$ & .712 & .191 & -.357 \\
\hline
\end{tabular}

$\mathrm{A}=0.283 f_{1}+0.833 f_{2}-0.056 f_{3} \quad$ (1)

$\mathrm{B}=0.275 f_{1}+0.671 f_{2}+0.195 f_{3}$

$\mathrm{C}=0.379 f_{1}+0.522 f_{2}+0.307 f_{3}$

$\mathrm{D}=0.566 f_{1}+0.444 f_{2}+0.244 f_{3}$

$\mathrm{E}=0.423 f_{1}+0.447 f_{2}+0.278 f_{3}$

$\mathrm{F}=0.202 f_{1}+0.460 f_{2}+0.580 f_{3}$

$\mathrm{G}=0.273 f_{1}+0.036 f_{2}+0.762 f_{3}$

(The rest of the data meaning is similar)

\subsubsection{Named Explanation of Factors}

Here, we orthogonalize the factor loading matrix using the Variance Maxima method to make the factors naming explanatory. Specify the factor loading in the descending order of the load of the first factor, and plot the factor load after rotation. 
Table 5: Rotated Component Matrix.

\begin{tabular}{|c|c|c|c|}
\hline & \multicolumn{3}{|c|}{ Ingredients } \\
\cline { 2 - 4 } & 1 & 2 & 3 \\
\hline $\mathrm{A}$ & .283 & .833 & -.056 \\
$\mathrm{~B}$ & .275 & .671 & .195 \\
$\mathrm{C}$ & .379 & .522 & .307 \\
$\mathrm{D}$ & .566 & .444 & .244 \\
$\mathrm{E}$ & .423 & .447 & .278 \\
$\mathrm{~F}$ & .202 & .460 & .580 \\
$\mathrm{G}$ & .273 & .036 & .762 \\
$\mathrm{H}$ & .497 & .470 & .268 \\
$\mathrm{I}$ & .513 & .204 & .369 \\
$\mathrm{~J}$ & .518 & .424 & .279 \\
$\mathrm{~K}$ & .702 & .382 & -.125 \\
$\mathrm{~L}$ & .326 & .645 & .274 \\
$\mathrm{M}$ & .054 & .703 & .472 \\
$\mathrm{~N}$ & .205 & .314 & .706 \\
$\mathrm{O}$ & .581 & .179 & .485 \\
$\mathrm{P}$ & .714 & .206 & .271 \\
$\mathrm{Q}$ & .736 & .158 & .323 \\
\hline
\end{tabular}

It can be seen from the table that "K provides enterprise technical support for R \& D products" and "P provides promotion channels for products or projects" has a higher load on the first factor. The first factor mainly explains these Variable, and can be interpreted as product development and promotion.

"A (The office space is adequate and the decor is comfortable) ", "M provides some financial support (or in-kind support) for the project" has a higher load on the second factor, and the second factor mainly explains these variables, which can be interpreted as economic support.

"G is the start-up business partner of the start-up team," "N is the source of funding for the project (investors and investment institutions)" and has a higher load on the third factor. The third factor mainly explains these variables, which can be interpreted as business information exchange.

\subsubsection{Calculate the Score of the Factor}

Here regression factor estimation factor score coefficient, and output factor score coefficient.

Table 6: Score coefficient matrix.

\begin{tabular}{|c|c|c|c|}
\hline \multirow{2}{*}{} & \multicolumn{3}{|c|}{ Ingredients } \\
\cline { 2 - 4 } & 1 & 2 & 3 \\
\hline $\mathrm{A}$ & -.334 & .269 & .207 \\
$\mathrm{~B}$ & -.066 & .086 & .095 \\
$\mathrm{C}$ & .111 & .196 & -.125 \\
$\mathrm{D}$ & .067 & .157 & -.096 \\
$\mathrm{E}$ & .107 & .174 & -.097 \\
$\mathrm{~F}$ & .123 & -.120 & .133 \\
$\mathrm{G}$ & .361 & -.163 & -.083 \\
$\mathrm{H}$ & .009 & .049 & .082 \\
$\mathrm{I}$ & .226 & .112 & -.208 \\
$\mathrm{~J}$ & .027 & .041 & .094 \\
$\mathrm{~K}$ & -.188 & .534 & -.196 \\
$\mathrm{~L}$ & -.238 & -.036 & .492 \\
$\mathrm{M}$ & -.067 & -.254 & .504 \\
$\mathrm{~N}$ & .277 & -.301 & .182 \\
$\mathrm{O}$ & .291 & -.014 & -.119 \\
$\mathrm{P}$ & .096 & .075 & -.049 \\
$\mathrm{Q}$ & .119 & .062 & -.076 \\
\hline
\end{tabular}


When calculating the value of two factor score variables, the total duration, the city's weight is higher, and the direction accords with the common sense, which is consistent with the actual meaning of the factor. In addition, the mean value of factor analysis scores was 3.47 and the standard deviation was 0.990 . Positive values indicate above average and negative values below average.

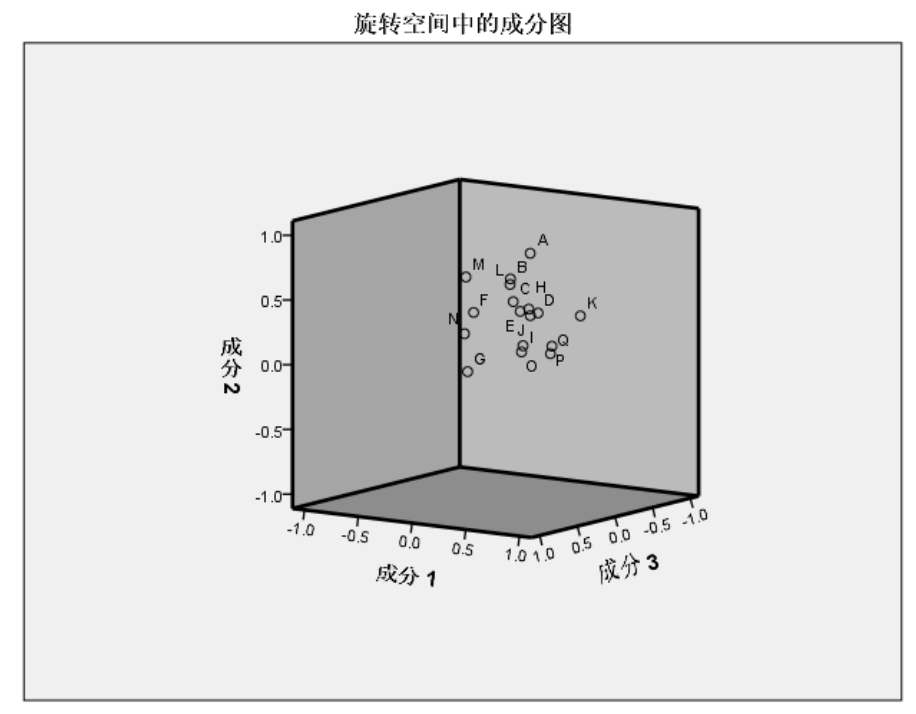

Figure 2: Ingredients figure.

\section{CONCLUSIONS AND SUGGESTIONS}

\subsection{Conclusions}

We found that the reason why entrepreneurs are willing to settle in co-working Space is mainly because they can enjoy the preferential policies of the country such as: ultra-low-cost settled and rent reduction; co-working Space will help small Business registration and tax account custody and other related procedures; co-working space for the maker to provide free open office space, office furniture; water and electricity free, free WIFI; free parking, rolling electronic screen free booths; provide free barber services ; Enjoy free library, gym; free conference room; enjoy the space to provide food subsidies, each meal as low as 6 yuan; free to enjoy the space to provide purified drinking water; daily free of charge the latest newspaper publications, real-time understanding of domestic and foreign news ; Provide free business counseling, policy solutions, project assessment, tax agents, personnel training, legal rights and other services; enjoy the space to provide investment and financing services; can apply for space seed funds and project special support funds

In recent years, the settled enterprises have also made remarkable achievements in development. Lvliang Netcom World Information Services Limited Company created a comprehensive large-scale Lvliang local "Public" platform - China Commodity Mall, now accommodating 300,000 online merchants, 500 transactions at the same time delivery, service up to 12 features, service medium and small 5500 micro-enterprises online at the same time docking, platform B2C, C2C Forum has been put into use.

\subsection{Suggestions}

\subsubsection{Diversified Development}

Co-working space functional needs should be more diverse, not only to office, but also social, to connect many resources and people. In other words, co-working space has the dual function of community and incubation.

In addition, a number of co-working space can also add some interesting elements based on the design characteristics of enterprises settled in the space to active and adjust the atmosphere. As an operator, how to provide management and start-up services for the settled enterprises is crucial. According to different stages of enterprises settled, the role operators play is not the same. In the second stage of entry, the operator is a business think tank, and should provide information 
positioning based on local policies and regulations, regional characteristics, professional data for enterprises, and provide good guidance to help enterprises do a good job of orientation and direction. (Huaxin Wang,2015).

In the second stage of entry, companies should design and decorate office space, then operators are responsible for the decoration in the role of master, providing enterprises with the design, construction and other integrated services.

\subsubsection{Subdivision Positioning to Adapt to Different Market Needs}

A successful co-working space should have the following characteristics: First, open and low cost. The so-called co-working space is to focus entrepreneurs on a common area, in which everyone brainstorm together, and this is the most basic condition is to achieve regional openness, and then can have the open mind. Entrepreneurship settled many are in the early days of entrepreneurship, so to provide a low-cost environment for them is very important. (Hui Zhang, Li Liu,2016)

Second, mutual assistance and cooperation. No matter what industry belongs to the settled enterprises, they should all be integrated with each other to become a good cooperative partner. co-working Space Operators should play a more role as a platform and bridge among them by organizing trade salons and training camps, Class competition, to enhance the degree of integration and cohesion.

Third, convenience, co-working space advantage should be reflected in the service, including the application for government grants, legal advice, business agents, in order to provide enterprises with a convenient business environment.

\section{REFERENCES}

[1] Changsheng He. Research on the Status Quo, Problems and Countermeasures of Co-working Space Development [J]. Innovation and Application of Science and Technology, 2016(24):283-283.

[2] Chunqing Wen, Junfang Jin, Xiaoyan Xu. Research on Co-working Space Development in China [J]. Cooperative economy and science and technology, 2016(13):118-119.

[3] Hongyi Dong. Research on the Problems and Countermeasures in the Co-working Space at Present Stage [J]. Science and Technology in Shanxi Province, 2016, 31(6):1-3.

[4] Huaxin Wang. Research on the Development and Supervision of "Co-working Space" [J]. China Market Regulation Research, 2015(10):53-55.

[5] Hui Zhang, Li Liu. Problems and Countermeasures of Co-working Space in Colleges and Universities in Hebei Province [J]. Managers, 2016(35). 